\title{
Mechanical properties and microphase structure of hydrogenated S-SB-S triblock copolymers
}

\author{
Yoshifumi Araki ${ }^{1}$, Daisuke Shimizu${ }^{1}$, Yukari Hori ${ }^{1}$, Kosuke Nakatani $^{1}$ and Hiromu Saito ${ }^{2}$ \\ We investigated the mechanical properties and microphase structure of a series of hydrogenated styrene- $b$-styrene-co-butadiene- \\ $b$-styrene triblock copolymers with various degrees of hydrogenation (DHs). The results indicated a distinct increase in the \\ tensile strength and modulus with increasing DHs. The results of dynamic mechanical analysis, transmission electron \\ microscopy and small-angle $x$-ray scattering revealed that the segregation of the microdomain structure strengthened with \\ increasing DHs. These results suggest that the enhancement of the mechanical properties with the DHs can be attributed \\ to an increased segregation of the microdomain structure, which physically cross-links points on the hydrogenated SB matrix. \\ The increased segregation of the microdomain structure can be attributed to the increased variation in the solubility \\ parameters of polystyrene and hydrogenated SB segments with increasing DHs. \\ Polymer Journal (2013) 45, 1140-1145; doi:10.1038/pj.2013.45; published online 15 May 2013
}

Keywords: block copolymer; hydrogenation; mechanical property; microphase structure

\section{INTRODUCTION}

Block copolymers have the ability to self-assemble into various ordered nanostructures, which are named microdomain structures. $^{1-2}$ ABA-type triblock copolymers consist of a pair of glassy outer blocks and a rubbery middle block, such as styrene$b$-butadiene-b-styrene (S-B-S) triblock copolymers. Spherical or cylindrical microdomains that are composed of hard thermoplastic segments act as cross-linking points of the soft-segment matrix. ${ }^{3-7}$ Block copolymers behave similarly to vulcanized rubber in deformation processes at ambient temperatures and can be meltprocessed at high temperatures by softening the hard domain. ${ }^{8}$

Although recent improvements in catalyst synthesis and polymerization techniques have enabled new living polymerizations, S-B-S is generally created through living alkyllithium-initiated polymerization, in which well-defined block copolymers are prepared by sequential monomer additions. ${ }^{9-12}$ S-B-S is employed in many applications, such as the manufacture of footwear, adhesives and compounded mechanical goods, as well as in asphalt modification. The mechanical properties of S-B-S are typically controlled by modifying the content of the polystyrene (PS) segment; that is, the tensile strength can be increased by increasing the content of the hard PS outer block segments. Owing to the presence of unsaturated hydrocarbons with double bonds in the polybutadiene middle block segments, S-B-S is vulnerable to thermal degradation. The weathering and thermal oxidative stabilities generally improve with hydrogenation of the polybutadiene middle block segments. The polybutadiene segments consist of poly(1,4-butadiene) and poly(1,2-butadiene). The hydrogenation of poly(1,4-butadiene) yields a polyethylene sequence, whereas the hydrogenation of poly(1,2-butadiene) yields a poly(1-butene) sequence. Hence, the hydrogenation of S-B-S yields styrene- $b$-ethylene-co-butylene- $b$ styrene (S-EB-S) triblock copolymers. Gergen et al. ${ }^{13}$ reported that the tensile strength of S-B-S increased after hydrogenation of all polybutadiene middle block segments.

A styrene- $b$-styrene-co-butadiene- $b$-styrene (S-SB-S) ABA-type triblock copolymer was also developed through living alkyllithiuminitiated polymerization. The S-SB-S triblock copolymer exhibits a low order-disorder transition temperature and strong shear-thinning behavior compared with the order-disorder transition temperature and shear-thinning behavior of the S-B-S triblock copolymer. ${ }^{14-17}$ Despite a styrene (St) content above $50 \mathrm{wt} \%$, hydrogenated S-SB-S triblock copolymer recently exhibited softness and flexibility, as well as excellent shock-absorbing, abrasion-resistant and filler-receptive properties. ${ }^{18}$ However, the effect of hydrogenation on the mechanical properties has not been clarified.

To understand the effect of hydrogenation on the mechanical properties, we prepared a series of hydrogenated S-SB-S triblock copolymers with various degrees of hydrogenation (DHs) for same St contents. The characteristic mechanical properties of the hydrogenated S-SB-S triblock copolymers were discussed in terms of the microstructures revealed by the results of dynamic mechanical analysis, transmission electron microscopy (TEM) observations and small-angle x-ray scattering (SAXS) measurements.

${ }^{1}$ Asahi Kasei Chemicals Corporation, Kawasaki, Japan and ${ }^{2}$ Department of Organic and Polymer Materials Chemistry, Tokyo University of Agriculture and Technology, Koganei-shi, Tokyo, Japan

Correspondence: Y Araki, Laboratory of Plastics, Asahi Kasei Chemicals Corporation, 1-3-1, Yakoh, Kawasaki-ku, Kawasaki-city, Kanagawa 210-0863, Japan.

E-mail: araki.yb@om.asahi-kasei.co.jp

Received 27 December 2012; revised 4 March 2013; accepted 7 March 2013; published online 15 May 2013 


\section{EXPERIMENTAL PROCEDURE}

The S-SB-S A-B-A-type triblock copolymer was synthesized through anionic polymerization using $n$-butyl lithium as an initiator in a cyclohexane solvent, as described by Yoshida and Friedrich. ${ }^{19}$ Commercially available monomers and solvents were purified using a typical routine. Polymerization was performed sequentially, that is, after the first PS block was polymerized by adding a St monomer; St monomer and butadiene one were added simultaneously at a constant rate for the SB middle block; St monomer was subsequently added to polymerize the PS outer block.

As butadiene monomer reacts more favorably to lithium chain ends than St, we carefully conducted the polymerization process for the SB middle block to maintain a constant St to butadiene ratio during the reaction. $N, N, N^{\prime}, \mathrm{N}^{\prime}$-tetramethylethylenediamine was added to the solution at the beginning of the process to achieve similar reactivities for both St and butadiene. The reactions were performed at $80^{\circ} \mathrm{C}$. To confirm the uniformity of the comonomer incorporation during the copolymerization, we extracted several samples from the reactor during the reaction and measured unreacted monomer concentrations with gas chromatography. We confirmed that the composition of the SB middle block during the reaction was within 3\% deviation.

The S-SB-S triblock copolymer consisted of two PS outer block segments and one SB middle block segment. The content of PS in the S-SB-S outer block segments and the content of St in the SB middle block segment are shown in
Table 1. The total content of 1,2-butadiene in the butadiene units is $20 \mathrm{wt} \%$. The average molecular weight of S-SB-S is 105000 .

The hydrogenation of S-SB-S triblock copolymer was performed using a titanocene catalyst in a cyclohexane solvent. Unsaturated hydrocarbons with double bonds of butadiene units in the SB block segment were hydrogenated. The butadiene unit in S-SB-S contains two isomers with double bonds, that is, 1,2-butadiene and 1,4-butadiene. Owing to the different reactivity of the double bonds of the two isomers, the hydrogenation of S-SB-S proceeded in a blocky fashion, as shown in Scheme 1. Because the double bonds of 1,2-butadiene are more reactive than the double bonds of 1,4-butadiene, poly (1,2-butadiene) was hydrogenated to yield a poly(1-butene) sequence and poly(1,4-butadiene) was hydrogenated to yield a polyethylene sequence. ${ }^{20}$

The hydrogenated S-SB-S was dried under vacuum at $60^{\circ} \mathrm{C}$ for 2 days. The characterization of the hydrogenated S-SB-S is shown in Table 1. Film with $2 \mathrm{~mm}$ thickness was prepared by press molding at $200^{\circ} \mathrm{C}$.

The stress-strain curves of the film specimens were obtained at room temperature using an Instron-type tensile-testing machine (VES05D, Toyo seiki Co., Ltd., Tokyo, Japan). The initial length and elongation rates were $40 \mathrm{~mm}$ and $500 \mathrm{~mm} \mathrm{~min}^{-1}$, respectively.

Dynamic mechanical analyses were conducted on a rheometer (ARES-G2, TA Instruments, New Castle, DE, USA) at temperatures ranging between -20 and $140{ }^{\circ} \mathrm{C}$, with a heating rate of $3{ }^{\circ} \mathrm{C} \mathrm{min}{ }^{-1}$ under a nitrogen atmosphere. The imposed strain and frequency were $0.5 \%$ and $1 \mathrm{~Hz}$, respectively.

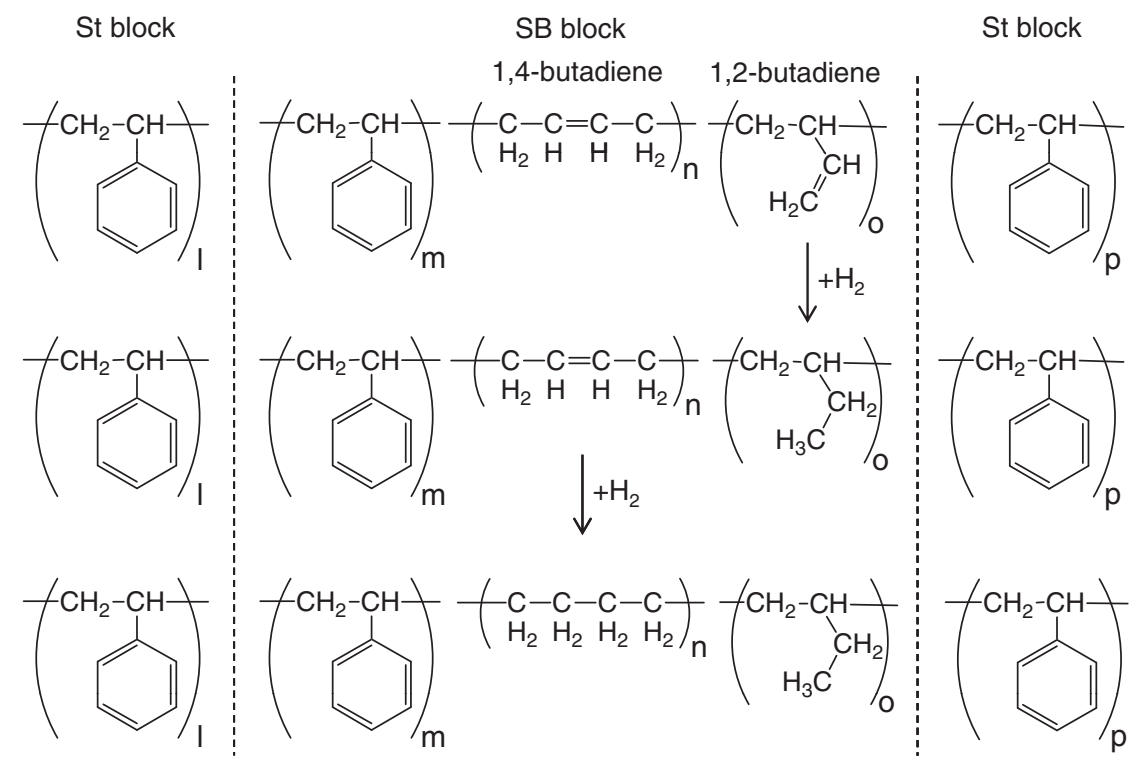

Scheme 1 Schematic flow of the hydrogenation of S-SB-S.

Table 1 Structures of hydrogenated S-SB-S

Structure of hydrogenated S-SB-S

Degree of

\begin{tabular}{lccccc}
$\begin{array}{l}\text { Molecular weight } \\
\text { Mn/Mw }\left(\times 10^{4}\right)\end{array}$ & $\begin{array}{c}\text { hydrogenation } \\
\text { in butadiene }(\mathrm{DHs})\end{array}$ & $\begin{array}{c}\text { Total St content } \\
\text { of outer PS blocks }\end{array}$ & $\begin{array}{c}\text { Total St content } \\
\text { of middle block }\end{array}$ & $\begin{array}{c}\text { Total St } \\
\text { content }\end{array}$ & $\begin{array}{c}\text { Schematic molecular structure of } \\
\text { hydrogenated S-SB-S }\end{array}$ \\
\hline 9.9/10.5 & $0 \%, 30 \%, 68 \%,>99 \%$ & $20 \mathrm{wt} \%$ (each 10 wt\%) & $47 \mathrm{wt} \%$ & $67 \mathrm{wt} \%$ & \\
\hline & & & & Outer PS blocks
\end{tabular}


For the TEM observations, ultrathin sections that were $\sim 70 \mathrm{~nm}$ thick were cut from the film specimen with a cryomicrotome. The section was stained by ruthenium tetroxide vapor for $5 \mathrm{~min}$ at room temperature. The morphology was observed by a transmission electron microscope (JEM 1230, Jeol Co., Ltd., Tokyo, Japan), with an acceleration voltage of $80 \mathrm{kV}$.

SAXS measurements were obtained using a NANO-Viewer system (Rigaku Co., Tokyo, Japan). $\mathrm{Cu}-\mathrm{K} \alpha$ radiation $(46 \mathrm{kV}, 60 \mathrm{~mA})$ was generated and collimated by a confocal max-flux mirror system (Rigaku Co.). The wavelength and the distance from the sample to the detector were $0.154 \mathrm{~nm}$ and $700 \mathrm{~mm}$, respectively. An imaging plate (Fujifilm BAS-SR 127, Fujifilm Co., Tokyo, Japan) was used as a two-dimensional detector, and the imaging plate reading device (R-AXIS Ds3, Rigaku Co.) was used to transform the obtained image to text data. The exposure time ranged from 2 to $4 \mathrm{~h}$, and the thickness of the specimens was $\sim 1 \mathrm{~mm}$. The scattering intensities were corrected with respect to the exposure time, the sample thickness and the transmittance.

\section{RESULTS AND DISCUSSION}

Stress-strain properties of a series of hydrogenated S-SB-S triblock copolymers with various DHs and equivalent PS contents are shown in Figure 1. The initial stress of the neat S-SB-S $(\mathrm{DHs}=0 \%)$ increased with increased strain in low-strain regions and then gradually increased to the breaking point at $\sim 550 \%$ of strain. Note that the tensile strength in Figure 1 distinctly increased after hydrogenation of S-SB-S. The modulus of the initial slope increased with increased DHs, whereas the strain at beak decreased with increased DHs. In the hydrogenated S-SB-S with high DHs, a sharp increase in stress was observed in high-strain regions and in low-strain regions after increasing the DHs. The stress at break for hydrogenated S-SB-S (DHs $>99 \%$ ) doubled the stress at break for neat S-SB-S $(\mathrm{DHs}=0 \%)$. The sharp increase in stress and the high strength at break point are behaviors similar to the behaviors of cross-linked rubbers. These results indicate that hydrogenated S-SB-S strengthens with increasing DHs, although the content of the hard PS outer block segment did not change and the soft SB middle block segment was not crystallized by hydrogenation. To understand the variation in stress-strain properties with the DHs, the phase morphology of a series of hydrogenated S-SB-S with various DHs is discussed in the following section.

Figure 2 illustrates the temperature dependence of the dynamic storage modulus $\left(\mathrm{G}^{\prime}\right)$ and dynamic loss tangent obtained by the dynamic mechanical analysis for a series of hydrogenated S-SB-S with various DHs. At $\sim 10{ }^{\circ} \mathrm{C}$, an inflection in the $\mathrm{G}^{\prime}$ curve and a peak in the dynamic loss tangent curve signifies the glass transition of the soft hydrogenated SB block segment. Conversely, an inflection in the G' curve at $\sim 100{ }^{\circ} \mathrm{C}$ signifies the glass transition of the hard PS block segment; flow occurs above this temperature.

The presence of the glass transition of the SB block segment and PS block segment indicates that the neat S-SB-S and hydrogenated S-SB-S comprise phases that separated as a result of the incompatibility between the PS and SB block segments. Although two separate $T_{\mathrm{g}} \mathrm{s}$ are sometimes considered a miscible blend because of their dynamic heterogeneity, ${ }^{21}$ the existence of the phase-separated structure was confirmed by TEM observations and SAXS profiles. The glass transition of the hydrogenated SB middle block segments occurred at higher temperatures and expanded with decreasing DHs. According to Kaplan, ${ }^{22}$ the glass transitions of the two components, which were estimated by the dynamic mechanical analysis, were similar and expanded as the size of the microdomain structure fell below $100 \mathrm{~nm}$, and each block segment was partially mixed. Thus, the results suggest that the PS and SB block segments were partially mixed during the hydrogenated SB-rich and PS-rich phases, respectively. That is, a moiety of PS segments mixed in the hydrogenated SB phase
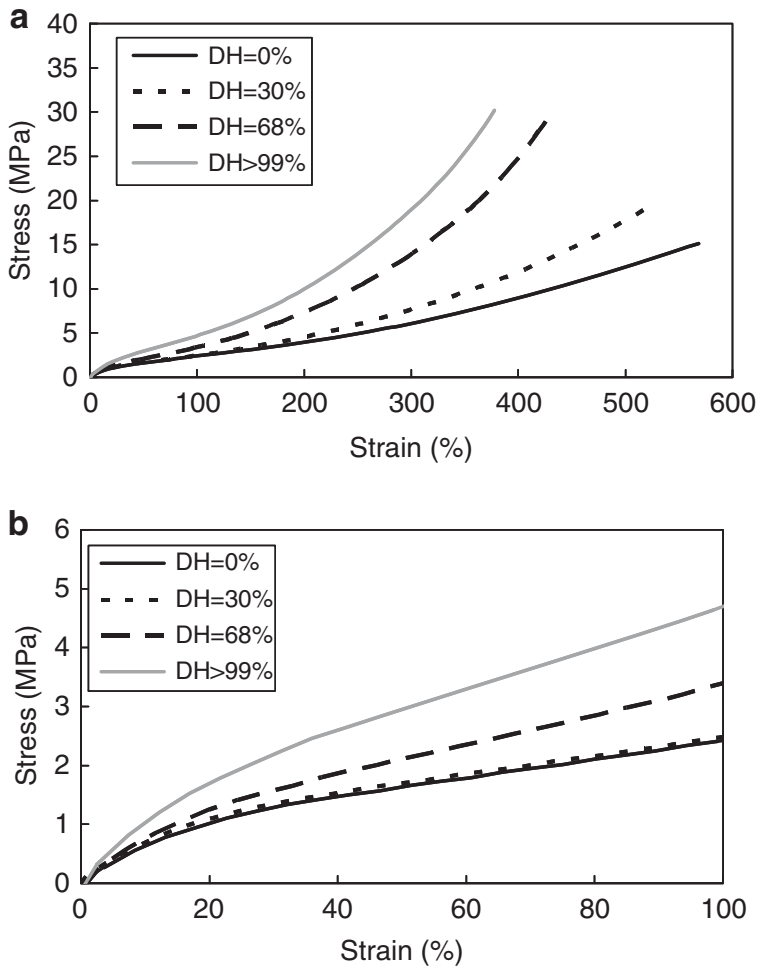

Figure 1 Stress-strain curves for hydrogenated S-SB-S. (a) high-strain region, (b) low-strain region.
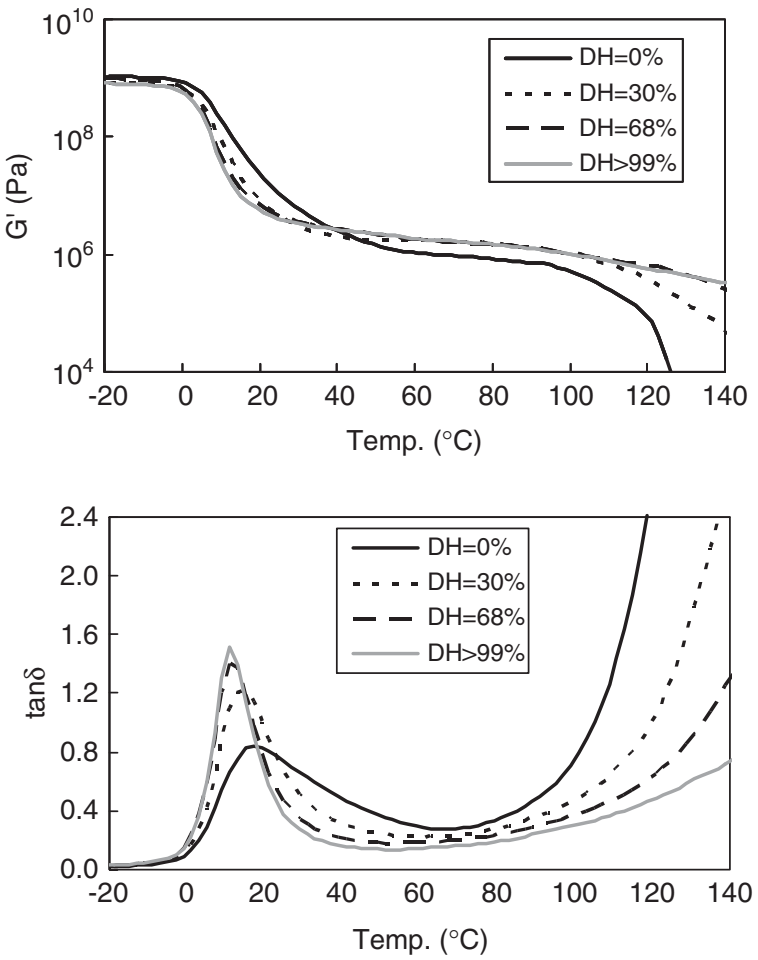

Figure 2 Dynamic mechanical analysis of hydrogenated S-SB-S. 
increased the glass transition temperature of the hydrogenated SB segment. With increased DHs, the glass transition temperature of the hydrogenated SB segment decreased and sharpened by increasing the incompatibility between the PS and hydrogenated SB block segments. Conversely, the G' value of the neat S-SB-S decreased at $\sim 100{ }^{\circ} \mathrm{C}$, and the decrease became more moderate with increasing DHs. This result suggests that the neat S-SB-S flows at $\sim 100{ }^{\circ} \mathrm{C}$, which is more suppressed because of an increased segregation of PS block segments, which resulted from an increased incompatibility with increasing DHs.

Figure 3 shows the TEM micrographs for a series of hydrogenated S-SB-S with various DHs. The structure is ambiguous in the neat S-SB-S (DHs $=0 \%)$. This observation supports the partial mixing of PS and SB block segments, as demonstrated by the dynamic mechanical analysis shown in Figure 2. Conversely, a sea-and-island microphase structure is observed in the hydrogenated S-SB-S. Given the low segregation power, the structure is undistinguishable compared with the microphase structure of typical microphase-separated block copolymers, such as styrene- $b$-isoprene and styrene- $b$-2-vinyl pyridine block copolymers. As ruthenium tetroxide preferentially stains St, the dark regions can be assigned to the hard PS block segments. Thus, hard PS block segments form the microdomains, whereas soft hydrogenated SB block segments form a continuous matrix phase. PS microdomains of $\sim 20 \mathrm{~nm}$ in diameter are uniformly dispersed throughout the continuous hydrogenated SB matrix. To examine the two-phase morphology, the SAXS results are explained quantitatively in the following section.

Figure 4 shows the SAXS profiles of a series of hydrogenated S-SB-S with various DHs. The scattering peak observed in the neat S-SB-S (DHs $=0 \%)$ was indistinct, indicating the absence of a clear twophase structure due to the partial mixing of PS and SB block segments, as suggested by the TEM observation in Figure $3 \mathrm{a}$. Conversely, a peak appeared in hydrogenated S-SB-S, indicating the presence of a two-phase structure with a periodic arrangement of PS microdomains. The peak shifted to the smaller angle region with increasing DHs. The correlation length between the nearest domains, $d$, can be calculated using Bragg's law $\left(d=2 \pi / q_{\max }\right)$, in which $q_{\max }$ is the scattering vector of the peak maximum. For instance, the calculated correlation length between the nearest domains in

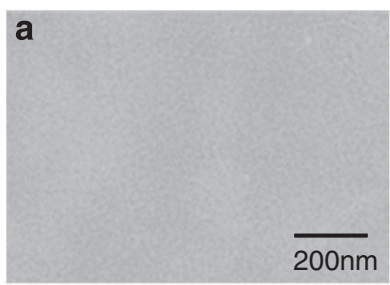

$\mathrm{DH}=0 \%$

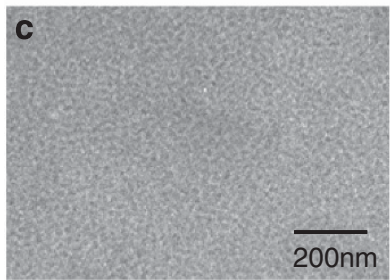

$\mathrm{DH}=68 \%$

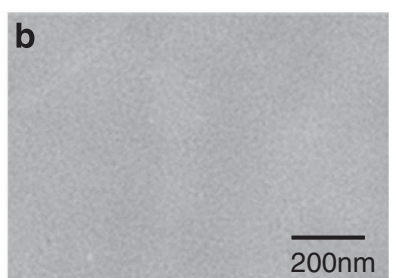

$\mathrm{DH}=30 \%$

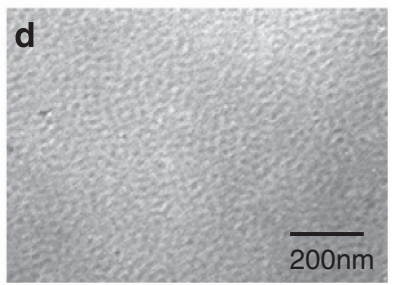

$\mathrm{DH}>99 \%$
Figure 3 Transmission electron microscopy (TEM) images of hydrogenated S-SB-S. (a) $\mathrm{DH}=0 \%$, (b) $\mathrm{DH}=30 \%$, (c) $\mathrm{DH}=68 \%$ and (d) $\mathrm{DH}>99 \%$. the hydrogenated S-SB-S (DHs $=30 \%)$ was $\sim 29 \mathrm{~nm}$ and the calculated correlation length between the nearest domains in the hydrogenated S-SB-S $(\mathrm{H}>99 \%)$ was $\sim 32 \mathrm{~nm}$. These results indicate that the microphase structure expands with increasing DHs owing to an increased incompatibility between the PS and hydrogenated SB block segments.

The most important result is that the SAXS peak intensity distinctly increased with increasing DHs, as shown in Figure 4. The peak intensity is related to the difference in electron densities of the PS domain and the hydrogenated SB matrix. As shown in Figure 5a, the peak intensity of the hydrogenated S-SB-S at low DHs was minimal because of the small difference in electron density, which can be attributed to partial mixing of the PS and hydrogenated SB block segments in the hydrogenated SB-rich and hydrogenated PS-rich phases, respectively. By increasing the DHs, the amount of the PS and hydrogenated SB block segments that are combined in the dissimilar block phase decreases, resulting in increased differences in electron densities and SAXS peak intensities, as schematically shown in Figure 5b. Thus, the SAXS results suggest that the segregation of the microphase structures strengthens with increased DHs owing to an increased incompatibility between the PS and hydrogenated SB block segments.

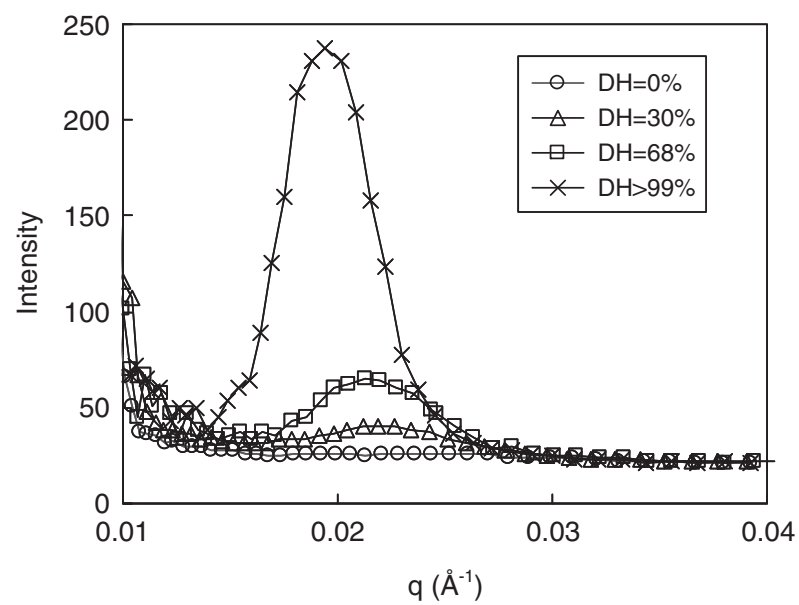

Figure 4 Small-angle $x$-ray scattering (SAXS) profiles of hydrogenated S-SB-S.
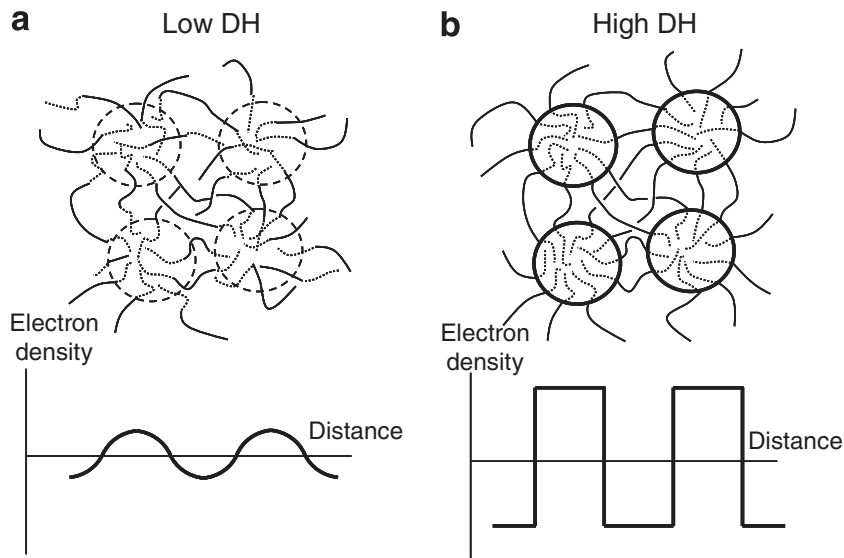

Figure 5 Schematic illustrations of phase-separated structure and electron density profile for hydrogenated S-SB-S with different DHs. 
The PS block microdomains are periodically dispersed throughout a continuous hydrogenated SB block matrix in the hydrogenated S-SB-S. The hard PS microdomains physically cross-link points on the continuous soft hydrogenated SB matrix. The cross-linking points, which consist of hard PS block segments, strengthen by decreasing the amount of soft hydrogenated SB segments in the PS microdomain and by increasing the segregation of the microphase structure. However, the cross-linking points are weak when the PS and hydrogenated SB block segments are partially mixed. Thus, the increased strength with increased DHs, as shown in Figure 1, is attributed to the increased segregation of the PS outer block segments and SB middle block segments, which is caused by the increased incompatibility between the PS and hydrogenated SB block segments with increasing DHs.

The incompatibility among the component segments in block copolymers can be illustrated by the Flory-Huggins interaction parameters of components 1 and $2, \chi_{12}$. The $\chi_{12}$ is given by

$$
\chi_{12}=M_{1}\left(\delta_{1}-\delta_{2}\right)^{2} / \rho_{1} R T
$$

where $M_{1}$ is the molecular weight of component $1 ; \delta_{1}$ and $\delta_{2}$ are the solubility parameters (SP values) of components 1 and 2, respectively; $\rho_{1}$ is the density of component 1 and $T$ is the temperature. As $\chi_{12}$

Table 2 Cohesive energy $(E)$, molar volume $(V$ and SP $(\delta)$ of homopolymers

\begin{tabular}{lccc}
\hline & $\mathrm{E}$ & $\mathrm{V}$ & $\delta$ \\
Polymer structure & $\left(\mathrm{J} \mathrm{mol}^{-1}\right)$ & $10^{-6}\left(\mathrm{~m}^{3} \mathrm{~mol}^{-1}\right)$ & $(\mathrm{MPa})^{1 / 2}$ \\
\hline Polystyrene & 36932 & 96.98 & 19.51 \\
Poly(1,2-butadiene) & 16450 & 58.26 & 16.80 \\
Poly(1,4-butadiene) & 18579 & 59.07 & 17.73 \\
Hydrogenated poly(1,2-butadiene) & 17527 & 65.62 & 16.34 \\
Hydrogenated poly(1,4-butadiene) & 18146 & 64.40 & 16.79 \\
\hline
\end{tabular}

Abbreviation: SP, solubility parameter. increases with the increase in incompatibility, the difference in the SP of components 1 and 2, $\Delta S P=\delta_{1}-\delta_{2}$, is a measure of incompatibility, that is, $\triangle S P$ increases as the incompatibility increases.

The SP $\delta$ is described by the cohesion energy $E$ and the molar volume of the polymers as follows:

$$
\delta=\sqrt{E / V}
$$

The SB block segment consists of random copolymers of St, 1,4-butadiene and 1,2-butadiene. The hydrogenation of the poly(1,4-butadiene) sequence yields polyethylene one, whereas the hydrogenation of poly(1,2-butadiene) yields poly(1-butene) one. The $E$ and $V$ of PS, poly(1,4-butadiene), poly(1,2-butadiene), hydrogenated poly(1,4-butadiene) and hydrogenated poly(1,2-butadiene) were calculated by Bicerano's method, ${ }^{23}$ considering the group contribution in the homopolymer; the calculated results are shown in Table 2.

As mentioned in the Experimental Procedure, the hydrogenation of S-SB-S proceeds in a blocky fashion, that is, the hydrogenation of poly(1,2-butadiene) occurs initially and the hydrogenation of poly(1,4-butadiene) occurs subsequently ${ }^{20}$ (Scheme 1); the 1,2butadiene units comprise $20 \mathrm{wt} \%$ of the total butadiene. Hence, the molar ratio of hydrogenated poly(1,2-butadiene) increases to $20 \mathrm{wt} \%$ by decreasing the molar ratio of poly(1,2-butadiene), and the molar ratio of hydrogenated poly(1,4-butadiene) increases to $80 \mathrm{wt} \%$ by decreasing the molar ratio of poly(1,4-butadiene). By following the previous hydrogenation procedure, the molar ratios of hydrogenated poly(1,2-butadiene) and hydrogenated poly(1,4-butadiene) for various DHs were determined, as shown in Table 3. The $E$ and $V$ of the hydrogenated SB block segments for various DHs were determined by the additive rule of $E$ and $V$ of the hydrogenated SB block segments of the repeat units shown in Table 2; the results obtained are listed in Table 3.

Table 3 also displays the SP $\delta$ of the hydrogenated SB block segments, which were calculated from equation (2) and the difference in the SP of the PS and hydrogenated SB block segments, $\triangle S P$, for various $\mathrm{DHs}$. The $\triangle S P$ increases with increasing $\mathrm{DHs}$, which indicates

Table 3 Amount of respective segments in the hydrogenated SB block; E, V and $\delta$ of the hydrogenated SEB block, and $\Delta$ SP between the PS

\begin{tabular}{|c|c|c|c|c|c|c|c|c|c|c|}
\hline & \multicolumn{6}{|c|}{ Amount of respective segments in hydrogenated SB block } & \multirow{3}{*}{$\begin{array}{c}E \\
\left(\mathrm{~J} \mathrm{~mol}^{-1}\right)\end{array}$} & \multirow{3}{*}{$\begin{array}{c}\mathrm{V} 10^{-6} \\
\left(\mathrm{~m}^{3} \mathrm{~mol}^{-1}\right)\end{array}$} & \multirow{3}{*}{$\begin{array}{c}\delta \\
\left(M P a^{1 / 2}\right)\end{array}$} & \multirow{3}{*}{$\begin{array}{c}\triangle \mathrm{SP} \\
\left(M P a^{1 / 2}\right)\end{array}$} \\
\hline & \multicolumn{3}{|c|}{ Non-hydrogenated unit } & \multicolumn{2}{|c|}{ Hydrogenated unit } & \multirow[b]{2}{*}{ Sum } & & & & \\
\hline & Styrene & $1,2-B d$ & $1,4-B d$ & $H-1,2-B d$ & $H-1,4-B d$ & & & & & \\
\hline \multicolumn{11}{|l|}{$\mathrm{DHs}=0 \%$} \\
\hline Weight ratio & 47 & 6.6 & 26.4 & 0 & 0 & 80 & 26134 & 75.09 & 18.66 & 0.86 \\
\hline Molar ratio & 0.425 & 0.115 & 0.460 & 0.000 & 0.000 & 1 & & & & \\
\hline \multicolumn{11}{|l|}{$\mathrm{DHs}=30 \%$} \\
\hline Weight ratio & 47 & 0 & 23.1 & 6.8 & 3.4 & 80.4 & 26215 & 76.18 & 18.55 & 0.96 \\
\hline Molar ratio & 0.425 & 0.000 & 0.402 & 0.115 & 0.057 & 1 & & & & \\
\hline \multicolumn{11}{|l|}{$\mathrm{DHs}=68 \%$} \\
\hline Weight ratio & 47 & 0 & 10.6 & 6.8 & 16.4 & 80.8 & 26139 & 77.40 & 18.38 & 1.13 \\
\hline Molar ratio & 0.425 & 0.000 & 0.185 & 0.115 & 0.275 & 1 & & & & \\
\hline \multicolumn{11}{|l|}{$\mathrm{DHs}>99 \%$} \\
\hline Weight ratio & 47 & 0 & 0 & 6.8 & 27.4 & 81.2 & 26059 & 78.38 & 18.23 & 1.28 \\
\hline Molar ratio & 0.425 & 0.000 & 0.000 & 0.115 & 0.460 & 1 & & & & \\
\hline
\end{tabular}
block and hydrogenated SB block

Abbreviations: $\mathrm{Bd}$, butadiene; $\mathrm{DHs}$, degrees of hydrogenation; $E$, cohesive energy; $\mathrm{H}$, hydrogenated; $\Delta S P$, .difference in solubility parameter; $V$, molar volume. 
that the incompatibility between the PS and hydrogenated SB block segments increases with increasing DHs. These results support the finding that an increased segregation of the microphase structure is due to the increased incompatibility between the PS and hydrogenated SB block segments with increasing DHs.

\section{CONCLUSION}

We determined that the tensile strengths and moduli of the hydrogenated S-SB-S triblock copolymers distinctly increased with increased DHs. The enhancement of the mechanical properties can be attributed to the increased segregation of the microphase structure with increasing DHs, owing to the increase in the incompatibility between the PS and hydrogenated SB block segments, as predicted by the difference in SP. Simultaneous measurements of stress and birefringence are powerful methods for understanding the deformation behavior of block copolymers with microphase structures. ${ }^{24-25}$ The origin of the characteristic deformation behavior of the hydrogenated S-SB-S will be explained by the simultaneous measurements of stress and birefringence in a forthcoming paper.

\section{ACKNOWLEDGEMENTS}

We thank H Shirai, Dr K Kato and Dr N Osaka for valuable discussions.

1 Molau, G. E. Block Copolymers Plenum, New York, 1970.

2 Matsen, M. W. \& Bates, F. S. Unifying weak and strong segregation block copolymer theories. Macromolecules 29, 1091-1098 (1996).

3 Hadjichristidis, N., Pispas, S. \& Floudas, G. Block Polymers (John Wiley \& Sons, Hoboken, 2003).

4 Honeker, C. C. \& Tomas, E. L. Impact of morphological orientation in determining mechanical properties in triblock copolymer systems. Chem. Mater. 8, 1702-1714 (1996).

5 Aggarwal, S. L. Structure and properties of block polymers and multiphase polymer systems: an overview of present status and future potential. Polymer (Guildf) 17, 938-956 (1976).

6 Sakurai, S. Progress in control of microdomain orientation in block copolymersefficiencies of various external fields. Polymer (Guildf) 49, 2781-2796 (2008).
7 Aoyagi, T., Honda, T. \& Doi, M. Microstructural study of mechaniacal properties of the ABA triblock copolymer using self-consistent field and molecular dynamics. J. Chem. Phys. 117, 8153-8161 (2002)

8 Sakurai, S., Okamoto, S. \& Sakurai, K. Developments in Block Copolymer Science and Technology. Melt Behaviour of Block Copolymers Ch. 4. John Wiley \& Sons, London, 2004) 127-158.

9 Quirk, R. P. Styrene-Diene Themoplastic Elastomers. Milestones and Recent development, 176th Technical meeting of the Rubber Division of the American Chemical Society 1981-1996 (2009).

10 Sugiyama, K. \& Hirao, A. Fundamental studies and recent advances in anionic polymerization. Nippon Gomu Kyokaishi 79, 543-549 (2006).

11 Webster, O. Living polymerization methods. Science 251, 887-893 (1991).

12 Hsief, H. L. \& Quirk, R. P. Anionic Polymerization, Principles and Practical Applications (Marcel Dekker Inc., New York, 1996).

13 Holden, G. \& Hansen, D. R. Thermoplastic Elastomers3rd EdnCh. 3Styrenic Thermoplastic Elastomers (Hanser, Munich, 2004).

14 Staudinger, U. et al. Enhancement of mechanical properties of triblock copolymers by random copolymer middle block. Euro. Polym. J. 43, 2750-2758 (2007).

$15 \mathrm{Knoll}, \mathrm{K}$. \& Niessner, N. Styrolux and styroflex from transparent high impact polystyrene to new thermoplastic elastomers. Macromol. Symp. 132, 231-243 (1998).

16 Lach, R., Weidisch, R. \& Knoll, K. Morphology and mechanical properties of binary triblock copolymer blends. J. Polym. Sci., Part B, Polym. Phys. 43, 429-438 (2005).

17 Ganb, M. et al. Influence of S/B middle block composition on the morphology and the mechanical response of polystyrene-poly(styrene-co-butadiene)-polystyrene triblock copolymers. Polymer (Guildf) 53, 2085-2098 (2012).

18 Sasagawa, M. et al. Hydrogenated copolymer and composition. US Patent 7964674 B2 (2011).

19 Yoshida, J. \& Friedrich, C. Thermorheological properties of hydrogenated pseudorandom styrene-butadiene copolymers. Macromolecules 38, 7164-7173 (2005).

20 Halasa, A. F., Massie, J. M. \& Ceresa, R. J. The Science and Technology of Rubber. The Chemical Modification of Polymers. 3rd EdnCh. 11. Elsevier Academic Press, Amsterdam, 2005) 497-528.

21 Lodge, T. P. \& McLeish, T. C. B. Self-concentrations and effective glass transition temperatures in polymer blends. Macromolecules 33, 5278-5284 (2000).

22 Kaplan, D. S. Structure property relationships in copoly- mers to composites. Molecular interpretation of the glass transition phenomenon. J. Appl. Polym. Sci. 20, 2615-2629 (1976).

23 Bicerano, J. Prediction of Polymer Properties. 3rd edn (Marcel Dekker, New York, 2002) 614-617.

24 Shimizu, K. \& Saito, H. Orientation of cylindrical microdomains of triblock copolymers by in situ stress-strain-birefringence measurements. J. Polym. Sci. Pt. B Polym. Phys. 47, 715-723 (2009).

25 Shimizu, K. \& Saito, $H$. Orientation relaxation of triblock copolymer with cylindrical microdomain by in situ stress-birefringence measurements. Polym. J. 41, 562-567 (2009). 